\title{
Complex Adaptive Systems and Comparative Politics: Modeling the Interaction between Institutions and Culture
}

\author{
Jenna Bednar ${ }^{1} \cdot$ Scott E. Page ${ }^{2}$
}

Published online: 9 August 2016

(C) Fudan University and Springer Science+Business Media Singapore 2016

\begin{abstract}
Comparative politics aims to explain political, economic, and social patterns within and across countries. Two approaches dominate and divide the field. Rational choice approaches explain differences in accountability, equality, corruption, and growth as a function of institutional features. Cultural and historical approaches rely on thick descriptive accounts that consider the complex interplay between behavior, formal rules, informal norms, identities, and the weight of tradition and history. In this paper, we describe how a complex systems approach might build a bridge between these schema by embedding lean rational choice models within contexts that include cultural, social, and historical detail and generating testable propositions about their interdependence. We first describe some fundamental elements of the complexity approach for comparative politics. As proof of concept, we next describe a research agenda of the effect of culture on institutional performance. Our framework considers multiple institutions in force both simultaneously and in sequence. It allows for behavior to depend on context, on the set of existing institutions, and on individuals' behavioral repertoires. In the spirit of rational choice models, human behavior will be purposeful. In the spirit of thicker, descriptive accounts, it will be contingent on time and place. It may be used to understand some instances of cultural exceptionalism and to understand the efficacy of economic and political development projects.
\end{abstract}

Jenna Bednar

jbednar@umich.edu

Scott E. Page

spage@umich.edu

1 Department of Political Science, University of Michigan, Ann Arbor and Santa Fe Institute, 426 Thompson Street, Ann Arbor, MI 48104, USA

2 Departments of Political Science and Economics and Center for the Study of Complex Systems, University of Michigan, Ann Arbor and Santa Fe Institute, 1085 S. University Ave., Ann Arbor, MI 48109, USA 
Keywords Comparative politics - Institutional design · Path dependence · Complexity $\cdot$ Robustness $\cdot$ Culture $\cdot$ Behavioral spillovers $\cdot$ Agent-based models

\section{Introduction}

One of the primary goals of comparative politics research is to understand why nation states vary in their ability to reach developmental targets. Countries differ in economic prosperity, in military aggressiveness, in the personal achievements of their citizens, and in their respect for human rights. They differ, in short, in the extent that they coordinate social activity for mutual benefit. Institutions of governance generate laws and policies (themselves institutions, but targeted to narrow domains) to shape behavior. So it is natural for comparativists to explore the role that institutions play in determining national outcomes.

The modern subfield of comparative politics has always been divided between area specialists and cross-country comparativists. Institutional analysis is similarly divided, between those who delve into the histories and cultures of particular places and those who construct models, whether empirical or mathematical, hoping to distil the essence of what causes political institutions to tend toward particular outcomes. Many within the subfield express concern for over this divide, dissatisfied with the relative poverty of each, and their apparent inability to learn from one another, despite intermittent efforts. ${ }^{1}$ Recently, two comparativists, Philippe Schmitter (2016) and Lane (2016) independently recommended complexity science as a bridge. $^{2}$

In this paper, we provide a brief primer on complex adaptive systems and then describe an ongoing research project that illustrates the potential of complexity science to contribute to questions within comparative politics. In that project, we apply ideas from complexity science to investigate the effect of cultural context on institutional performance. Our approach assumes purposive actors embedded in multiple institutions and then builds toward thick description.

What follows consists of three parts followed by a brief discussion. In the first, we describe the research challenge: how can institutional analysis account for the effect of culture dynamically, where both institutions and culture adapt in response to one another? We introduce our main idea: that institutions can produce different outcomes in different cultural contexts because of the spillover of behaviors between institutions. These spillovers imply that institutional designs must take existing behavioral patterns into account and that evaluating institutions in isolation ignores the sway of the past and the existing context.

In the second part, we summarize theoretical insights from complexity science that we believe are most relevant to institutional performance and design. Thirty

\footnotetext{
1 Two projects stand out: the current work by historical institutionalists to create a methodology that can lead to portable theories (see, eg. Mahoney and Thelen 2009), and the analytic narratives project (Bates et al. 1998), a group of rational choice modelers who turn to history to raise questions, develop models to explain unexpected twists, and then use history to support the predictions of the model.

${ }^{2}$ Nearly two decades ago Robert Jervis (1998) made the same recommendation for international relations.
} 
years ago, a scholar could say "it's complex" and be absolved of any obligation to constrain the possible implications. That is no longer true. Complexity science has developed as a field with theory, models, measures, concepts, norms, and properties. To define a system as complex now implies regularities and results in a way that it did not in the past. For example, we discuss how the same system can produce an equilibrium or complexity depending on initial conditions and the path followed (Wolfram 2002). This finding reveals a false distinction between equilibrium systems and complex systems. The same system, or in our case, institution, could produce either or both types of outcomes.

Central to our analysis will be that outcomes in complex systems emerge from the bottom up. We will not assume our result. The complexity of the dynamics will depend on how actors learn, the amount of interdependence, the diversity of the system, and the network structure (Page 2010). Last, we discuss how system robustness, the capacity to maintain functionality, differs from game-theoretic notions of equilibrium stability.

In the third part, we introduce a method for analyzing the effect of culture on the capacity of institutions to shape behavior. Our framework defines a cultural context as an ensemble of institutional settings along with the patterns of behavior that result from those institutions. Within a cultural context, purposive individuals develop behavioral repertoires that they use to respond to institutions. The repertoires that emerge exhibit consistency across institutions; actors apply similar rules in related settings. In our models, as in case studies, evolved patterns of behavior may outlive the institutions that created them and influence the performance of existing institutions and the choice over future institutions.

\section{The Puzzle of Cultural Context}

That institutions should matter, i.e., that equality, growth, corruption, accountability, and representativeness should depend on human choices as guided by institutional features, seems indisputable and has been a persistent thesis of comparative political science, from Aristotle to Acemoglu. The primacy of institutional features as a cause of variation is less clear. A second factor, culture, competes with institutions to explain outcomes. To understand the effect of institutions and of culture, one might treat them as independent variables, and somehow sum up their joint contribution to outcomes. Another approach-the one we will advocate-is to treat culture and institutions as mutually constructive, as affecting one another. Like Petri dishes in a lab experiment, culture is an environment that can affect the way that institutions shape behavior. The cultural context does not remain unchanged with the addition of institutions, but can be transformed. Understanding the effect of either on behavior requires a theory of how they interact.

By definition, comparative politics is interested in the effect of cultural context, whether the methodology is quantitative or qualitative. Comparative institutional analysis has long been embedded in the rich context of area studies: characterized by close observation of current events, rich interpretations of documents and events, 
interviews of key actors, and analysis of data gathered from surveys and government reports whose meaning becomes apparent within the context of a historical and cultural narrative. Around a half-century ago, with the rise of the behavioral political science and accompanying advances in empirical measurement and analysis, large-n studies became possible. Institutional effects were captured in broad strokes by cross-country comparativists who looked for general patterns; many a scholar considers Lijphart (1984 or 1999) an indispensable reference on the effects of institutions. When dummy variables stand in for regions, or ethnic communities, or language groups, the analyst tips a hat to the significance of culture. In either approach, the study of cultural context and institutional features are inseparable; each is necessary for the story to be fully told.

Empirically oriented political science has raised provocative correlations about the relationship between culture, institutions, and outcomes, but the inductive nature of the work means that the focus is not on identification of mechanisms. ${ }^{3}$ The introduction of rational choice models into comparative politics analyzed institutional effects not in terms of the evidence, but from the other end: deductively, reasoning from behavioral axioms and problem-specific working assumptions to generate hypotheses about the way that human behavior is shaped by institutions, given the information and incentives that the institutions provide. The rational choice approach offered the possibility of a core set of models that could explain patterns of equality, economic growth, corruption, and war-making within and across countries, captured not in terms of statistical correlations or unique histories, but through the choices that humans make. ${ }^{4}$

In rational choice models, context, culture, and history matter in games with multiple equilibria. Among these games, one can distinguish between two types. In pure coordination games, such as choosing how to greet one another, whether to wear shoes indoors, or whether to write from left to right or right to left, equilibria have identical payoffs a priori. Of course over time, coordinated equilibria such as ritual celebrations of births, marriages, and death do take on cultural significance. In these games, a game theorist might invoke culture to break ties in a model's prediction. It can explain variation without abandoning any rationality assumptions.

In all other games with multiple equilibria, culture must exert more force as it is directing outcomes that advantage some at the expense of others or in some cases directing societies to inefficient outcomes. Culture might bias outcomes toward equality, efficiency, or even risk aversion. Qualitative scholars deride this more expansive use of culture as a selection mechanism because it abandons the principal of behavioral universality and allows game theorists to choose whichever equilibrium best fits the data.

As an example of the advances and limitations of existing comparative analysis of culture and institutions, consider data from a meta-analysis of thirty-seven experiments of the Ultimatum Game, spanning 25 countries (Oosterbeek et al.

\footnotetext{
${ }^{3}$ An important exception is the movement in historical sociology of process tracing as a method to identify causal mechanisms within specific cases. See, for example, Thelen (1999), Mahoney and Thelen (2009), Falleti and Lynch (2009).

${ }^{4}$ The game-theoretic framework is becoming a standard approach to teaching undergraduates: see Clark et al. (2012)'s textbook.
} 
2004). In the Ultimatum Game, one player makes a take-it-or-leave-it offer of a split of some amount of money and a second player can accept or reject the offer. If the second player accepts the offer, the two players receive the amounts proposed by the first player. If the second player rejects, both players receive zero. The subgame perfect Nash equilibrium of this game calls for the first player to offer the smallest possible amount to the second player, which the second player should accept as it is better than nothing. However, any split in which both players receive non-negative amounts of money can also be an equilibrium. ${ }^{5}$

Figure 1 plots the initial offers scaled between 0 and $100 \%$ for 13 countries (none were above $50 \%$ ) against their scores on this factor. The data reveal substantial variation: average offers in the Ultimatum Game (along the $Y$-axis) range from $25 \%$ in Peru to $45 \%$ in Japan. ${ }^{6}$ The data violates the standard game-theoretic null assumption of no cultural variation in offers. Oosterbeek et al. (2004) show that scores on the Traditional/Secular-Rational dimension from the World Values Survey ( $x$-axis) negatively correlate with offers. ${ }^{7}$

This particular regression is not a test of a theoretical hypothesis; it shows an interesting correlation that encourages more questions. Oosterbeek et al. (2004) tried several cultural variables (including degree of Post-Material values from the World Values Survey). Only the secular-rational score correlates significantly with offers. That fit, however, is far from perfect: the United States lies well above the regression line, Spain lies well below and Japan and Sweden load similarly yet their initial offers differ by a large amount. Even if we ignore the substantial outliers, we still have no real explanation for why the offers differ. The regression shows that survey responses correlate with experimental results. It does not provide any insight into how history, culture, or institutions might influence behavior.

In a different study of some of the same games as in the meta-analysis, Henrich and his colleagues - a collaboration between economists and anthropologists-link behavior to the broader institutional setting. They investigate connections between the offers and the larger economic, culture, and social contexts of each society. They found that average first round offers increase in interdependence in economic production, market integration, and the extent of gift-giving norms. For example, the Lamalera, Indonesians who collectively hunt whales, made high offers. The Machiguenga, who rarely cooperate outside their families, offered the least.

Henrich et al. (2001) find that the way that people behave in one setting-a laboratory experiment-appears to be influenced by their behaviors in other institutions: markets, economic production, and gift-giving norms. In Sect. 4, we will refer to these patterns as behavioral spillovers and identify them as cultural hallmarks. Behavioral spillovers are also evident in offer rejections. Rational

\footnotetext{
${ }^{5}$ Suppose that the first player must offer a split of ten dollars. To see how the first player getting six dollars can be a Nash equilibrium, we need to only assume that the second player only accepts offers of four dollars or more. If the first player offers four dollars, then neither player can benefit by changing strategies.

${ }^{6}$ There exists a growing literature in both psychology and economics that explores cultural differences in behavior. See for example Nisbett et al. (2001) or Medin et al. (2010).

${ }^{7}$ Oosterbeek et al. invert the world values survey measure. Here, higher numbers correspond to a greater respect for authority.
} 


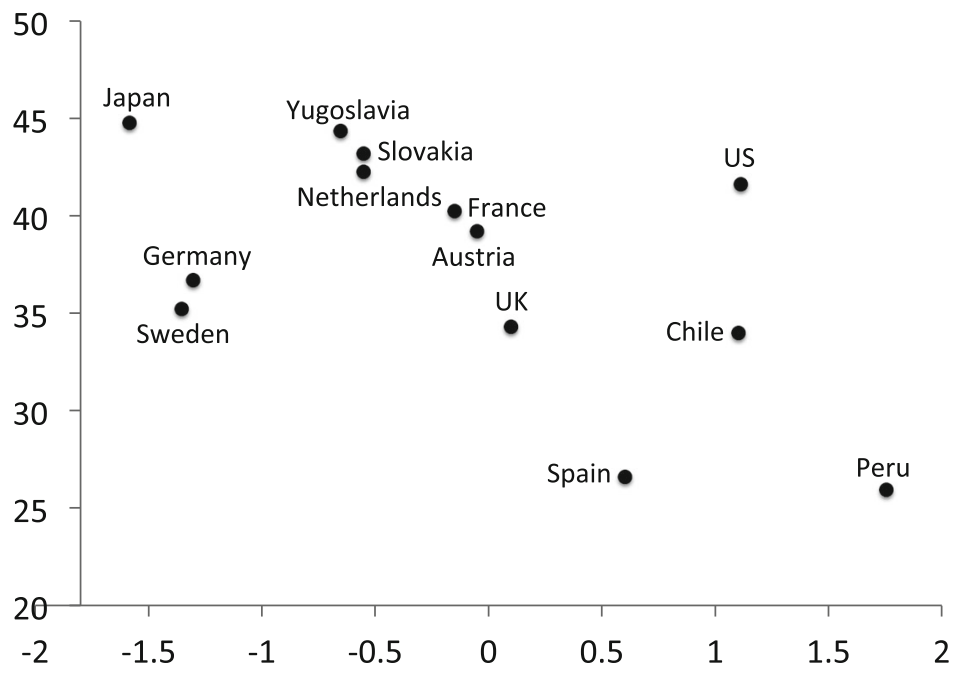

Fig. 1 Initial offers in the Ultimatum Game and World Values Survey Traditional/Secular-Rational scores, created by authors using data from Oosterbeek et al. (2004)

choice theory classifies rejecting any offer as illogical. Rejecting a high offer is especially irrational. And yet, subjects from cultures with strong reciprocity norms often refused high offers. The did so to avoid an implicit future debt. A prevalent behavior in other settings-reciprocity-bled over into the experiment. In a companion set of public good experiments, Henrich et al. (2001) found more evidence of behavioral spillovers. Upon hearing the instructions, the Orma referred to the experiment as a harambee: an institution of voluntary contributions to local projects. The Orma subjects then contributed at a high level as they would for roads and schools.

One can model spillovers across institutions by changing payoffs. For this to happen, the existence of one game must change the payoff in another game. There are three ways to do this (and we will introduce a fourth). First, games can be linked, where payoffs of one game are determined by play in another, such as in Putnam (1988)'s model of domestic audiences driving diplomacy, or in Tsebelis (1990) model of comparative party politics. The modeling applications are not particularly focused on culture, but the method allows it. Second, payoffs may change exogenously, in response to other, unmodeled, social changes. Ferejohn (1991) adopts this approach in describing electoral transfers of power in British parliamentary history. In his model, a change in norms shifts payoffs to allow a new equilibrium to be enforced. Ferejohn substantiates his assumption of a change in payoff with historical evidence of a change to the political culture. ${ }^{8}$ Critics of this approach point out that by an appropriate change of payoffs, a modeler could make any outcome an equilibrium.

Third, payoffs may change as the game is played. Greif (2006) and Greif and Laitin (2004) model quasi-parameters: consequences of outcomes that affect future payoffs. In their model, an institution either positively or negatively reinforces

\footnotetext{
${ }^{8}$ See also the analytic narratives project of Bates et al. (1998).
} 
itself. This could occur because it creates subsidiary institutions that support or undermine the institution in question. Culture is captured by the beliefs of agents subject to the institution; beliefs are slow to change, and may fail to update as game payoffs change. This approach enables them to show how some institutions can be self-stabilizing and others can produce endogenous change.

While we do not dispute that changes in payoffs can occur, we do not think this captures all cases. In particular, it says little about how behavior in one setting was transferred to another setting, as was the case in the experiments by Henrich et al. Culture refers to more than a single kind of encounter. It implies that responses to distinct games are somehow related to one another, even if the games are not explicitly linked through their institutional form.

We offer a different approach to the effect of context, looking at how institutions can build patterns of behavior that spill over from the bounds of one institution to affect an agent's response in another. Individuals interact repeatedly in multiple institutions and possess behavioral repertoires. Initial actions to any new institution depend on the behavioral repertoire. The agents then learn what to play in response to what other members of the population play. The long run behavior that emerges can depend on the initial behavior, on play along the path, i.e., be path dependent, or be independent of both. These can be thought of as the regular patterns of reactions that constitute a part of the social fabric that allows us to characterize societies broadly.

NNotice that we speak of outcomes emerging as opposed to equilibria being selected or reinforced. We do so because we adopt a complex systems approach in which outcomes emerge from the bottom up through the interactions of the actors. In our framework, the behavioral repertoires, like the set of actors and the payoffs, comprise the initial conditions of the model. We do not presume an equilibrium, nor do we presume that everyone eventually adopts the same behavior. In fact, that rarely occurs. Instead, the population of actors evolve a heterogeneous collection of behaviors.

We have constructed a model of culture where we identify specifically how culture interacts with institutional incentives and information to shape behavior. Our approach aligns with Swidler's toolkit model of culture. In her view and ours, culture guides strategies of action-" "persistent ways of ordering action through time“ (Swidler 1986:273). Our more formal implementation relies on an evolutionary model in which people build behavioral repertoires as they interact within institutions. It shares features with Boyd and Richerson's (1988, 2005) pioneering work on cultural evolution in which culture is a socially-transmitted cognitive construct. We differ in that Boyd and Richerson conceive of culture as being information stored in the heads of agents, while for us, culture is an emergent feature of a community. In our model, each agent possesses a collection of behavioral rules that they apply in their daily interactions. No two people will likely have the same exact set. Yet, within a community, people will share many behaviors resulting in a heterogeneous coherence of repertoires. ${ }^{9}$

\footnotetext{
9 Lizardo and Strand (2010) provide an excellent overview of the toolkit model, its close cousin practice theory, and their distinctions from the more classical models of socialization. The latter imply a highly coherent, well-formed understanding of codes and value systems. Our model suggests a more experiential cognitive process and embraces diversity with cultures that is more difficult to sustain with classical theories.
} 


\section{Complexity and Complex Systems}

To lay the groundwork for the complex system-based framework of institutional spillovers that we present in the next section, here we provide a brief primer on complexity theory. For the most part, we restrict attention to those aspects of complexity theory with immediate relevance for comparative political analysis. We show that complex systems approaches offer the potential to exhibit many of the desiderata expressed by comparativists like Schmitter (2009) and Lane (2016).

We begin by defining complexity, listing the essential elements of complex adaptive systems, and distinguishing complexity from "complicated". ${ }^{10}$ We then note five characteristics of complex systems salient for understanding the problem noted in Sect. 2: how the same institution could generate different responses in different contexts.

A system exhibits complexity if it is difficult to describe, explain, engineer, evolve, or predict. Complex phenomena lie between ordered patterns and random sequences (Page 2010). Systems capable of producing these phenomena, i.e., complex systems, consist of diverse entities or actors that follow behavioral rules. These actors are situated within networks - their location and relationship to one another matter-and their behavioral responses depend on the actions of others. The situated interactions combine to meso- and macro-level patterns.

Many political and economic systems possess these defining characteristics: (a) diverse agents that (b) interact in space and time who (c) adaptively respond to the actions of others. The phenomena that emerge from these systems are often complex in the statistical sense. The time series of stock market prices or oil prices serve as illustrative examples. Neither can be easily explained or described. Adjusting for trends, stock prices cannot be predicted, and yet patterns do exist. Prices are best described as somewhere between random and as a predictable pattern. The same might be said of time series of international alliances and political opinion: they are neither predictable patterns nor random. They are complex.

When a system produces a complex sequence of outcomes, individual connections and behaviors respond and react to produce non-stationarity-the mean response and the variation in responses both change over time. Behaviors adapt. These adaptations distinguish "complexity" from "complicated". For example, the diverse parts in a complicated watch follow fixed rules. The parts do not respond to the watch's macro behavior of telling time. In contrast, the parts of an electoral system do respond. Voters, candidates, and parties engage in an elaborate dance of adaptations as polls are released or electoral returns announced. Watches are complicated, electoral systems are complex.

Although system complexity is often invoked metaphorically-e.g., "markets are complex" or "politics are complex"-the complexity of a system can also be analyzed scientifically. Complexity science has advanced significantly over the past thirty years. Thousands of scientific papers have transformed complexity theory

\footnotetext{
${ }^{10}$ For further study, we suggest Miller and Page (2007) and Mitchell (2009), and online resources including the MOOCs available on the Santa Fe Institute's education site (https://complexityexplorer. org), Scott Page's MOOC "Model Thinking" at https:/www.coursera.org/learn/model-thinking, or "Understanding Complexity" at www.thegreatcourses.com/courses/understanding-complexity.html.
} 
from a collection of provocative insights (e.g., "more is different," "the edge of chaos") into a coherent discipline (Miller 2016). For example, early complexity research consisted of examples of systems that produced long-tailed distributions. The literature now contains multiple formal models to derive the shapes and slopes of those distributions. ${ }^{11}$ An early paper in complex systems might demonstrate how two network structures produced different outcomes. The current state of the art includes theoretical explanations for how outcomes depend on degree distributions, betweenness measures, and path lengths (Jackson 2008; Newman 2010).

Only a small portion of the developments in complexity theory will be germane to comparative politics and an even smaller set will be central to the present analysis. The literature on long-tailed distributions provides one example of a set of findings relevant to comparative politics generally but not to institutions specifically. Models of self-organized criticality produce long-tailed distributions, i.e., large events, but they cannot predict when such events occur. Thus, complex system scholars would claim that their models provide insights into why the distribution of war deaths, terrorist acts, and political uprisings have long tails (Johnson 2009), but would hasten to add that they cannot predict singular events, such as the dissolution of the Soviet Union or the selection of Donald Trump as the U.S. Republican Party's presidential nominee.

We focus here on five insights from complex systems relevant for understanding cultural context how the same institution can produce distinct outcomes in different settings. The set, listed in the box on page 14, includes classes of outcomes, system properties, and relationships between properties and outcomes.

First, complex systems can produce four classes of outcomes: equilibria, patterns and cycles, randomness/chaos, or complexity (Wolfram 2002). Equilibrium-the focus of nearly all game-theoretic analyses-occupies no special place in complex systems theory. As we describe in a moment, a single system might exhibit any one of the classes of outcomes depending on conditions. Furthermore, a single system may produce multiple outcomes belonging to different classes.

Oil markets again prove illustrative (Page 2015), with the two key outcomes, prices and production, falling into separate output classes. Output follows a regular pattern: a linear increase. The sequence of oil prices is complex. By looking at features of the market, we can see how this comes to be.

The world oil market consists of producers and consumers scattered across hundreds of countries. Some actors belong to cartels that limit production. Capacity constraints on refining and delivery influence prices as do political considerations.

\footnotetext{
11 These long-tailed distributions, unlike normal distributions, have multiple causes including positive feedbacks, self-organized criticality, and random walk return times. Under positive feedbacks, people choose to live where other people live, cite papers that other people cite, and buy products that other people buy. This results in distributions of city sizes, paper citations, and some products sales with long tails. In the self-organized criticality model, tension within a system builds at a regular rate. Relaxation (tension-release) events, such as earthquakes, avalanches or market crashes, exhibit a power law distribution. Finally, in a random walk, a process starts at zero moves up or down each period. If we keep track of the number of periods between return times to zero, that will be a long tail. If we think of the population size of some group as following a random walk, this model can explain why the distribution of lives of firms, organizations, and species have long tails. See Newman (2005) for the explicit derivation of power laws.
} 


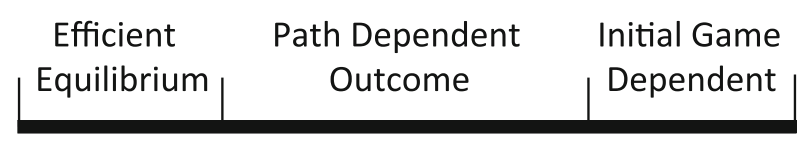

\section{Extent of Behavioral Spillover}

Fig. 2 How type of equilibrium depends on extent of spillover

The oil market has all the attributes of a complex system: diverse actors that interact locally by following rules. The actors produce macro phenomena, and those phenomena influence how actors behave. The system produces a complex time series of oil prices: prices are difficult to explain, describe, or predict. The global oil market also produces regular patterns. The time series of global output of oil is not complex. It grows at a steady rate with an occasional drop due to wars in the middle east. The regularity of global oil output arises from standard economic logic: the supply rises to meet the global demand which tracks global economic growth.

Second, complex systems models are rule based and bottom up. Actors are socially situated and follow and adapt rules of behavior. These behaviors aggregate to produce outcomes. For this reason, Epstein (2006) refers to the complex systems approach as generative. The modeler assumes behaviors and describes the context and lets the model run. In game theory models, the modeler assumes payoffs and beliefs and derives the outcome. Game theorists must construct assumptions with an eye on tractability. Complex system modelers confront no such constraint. Some phenomena that emerge in the models they build may turn out to be amenable to mathematical analysis. If so, the mathematics often provides alternative insights into the causes of those phenomena.

Third, the outcome that does emerge, including the dynamic class of that outcome, can depend on initial conditions as well as on the path of responses. Any contextual effect, including culture, therefore, determines not only which outcome occurs but whether the system attains an equilibrium or is complex. Figure 2 summarizes analytic results derived in Bednar and Page (2015). It shows how the choice of equilibrium can depend on context. In that paper, described in more detail below, agent behavior in one game spills over to affect responses in another game. The extent of the spillover is variable. As it increases, outcomes shift from being equilibria to depending on the sequence that the institutions are introduced to the system - institutional path dependence. As the spillovers become large, the system's outcome depends on the initial game-the so-called "butterfly effect." Path dependence poses problems for comparative static analyses; a new run of the model may generate a different path, causing the analyst to arrive at a different conclusion about the relationship between variables.

Figure 3 graphically represents how the class of outcome might vary with the rate of adaptation and the level of diversity. For low rates of adaptation and diversity, the system might reach an equilibrium. As the rate of adaptation increases, the system might display order, perhaps by producing cycles. If diversity also increases, the 


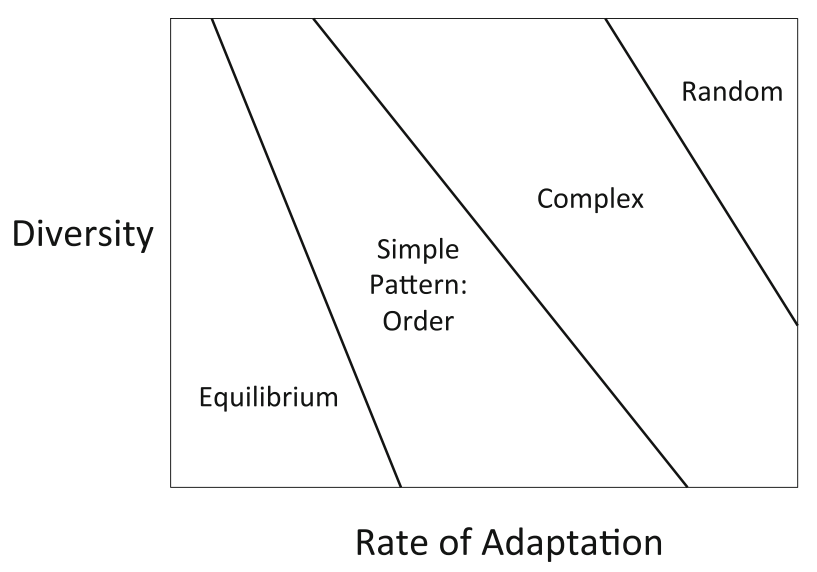

Fig. 3 Outcome class as a function of diversity and rate of adaptation

system may produce complex patterns. If both diversity and the rate of adaptation are high, the system may produce randomness.

Fourth, as with rates of adaptation and levels of diversity, the number of connections (interconnectedness) and the magnitude of the interactions among actors (interdependence) also contribute to whether a system exhibits complexity. The connection between interdependence and complexity is not unidirectional; complexity occurs at moderate levels of interdependence (Miller and Page 2007). With limited interactions such as in summing or averaging, a central limit theorem logic holds. Aggregate phenomena will be predictable and the system will exhibit no complexity. With too much interdependence every action depends in a complicated way on every other action. Actors within the system lack the capacity to produce and exploit structures and randomness ensues.

At moderate levels of interdependence, changes in one actor's behavior create mild echoes through the system, causing a few other actors to change their behavior. These interactions keep the system churning. They maintain novelty and produce path-dependent outcomes. A change in behavior at a particular moment in time can produce a cascade of responses. Different behaviors on the path can produce different responses, implying that the path can matter.

Interdependence correlates with connectedness, although not perfectly. Actors can be connected, but the extent of interdependence can be small. This occurs in commodity markets. Each purchaser's demand contributes in only a small way to the market price. The market may well reach equilibrium. Alternatively, a political candidate in a primary may be connected to only a handful of other candidates, but each action may have a large effect. As a rule of thumb, more connectedness increases complexity to a point and then complexity can fall if actors base their behavior on averages.

Last, complex systems scholars distinguish robustness from stability. Stability means to return to the same point. Systems with a single stable equilibrium return to 
the same configuration after a perturbation. Robustness refers to the capacity to maintain functionality. A system can be robust to knockout, invasion, internal dynamics, or changes in the external environment. A culture may be robust to the introduction of new institutions or new actors. Within a robust democracy, when a political party collapses or a new party forms, the democracy continues to function, albeit differently. In federal systems, the distribution of authority between national and state governments may change in response to actor's behaviors, but the essence of the system, the agents and the safeguards, remain unchanged. The adjustments to the distribution may be the key to federal system robustness (Bednar 2009).

\section{Five Complex Systems Insights Relevant to Comparative Institutional Performance}

1. Complex systems can produce outcomes in four classes: equilibria, simple patterns, complex patterns, or random. The same system might produce multiple classes of outcomes.

2. Outcomes emerge in complex systems from the bottom-up. Actors follow and adapt purposive rules. Aggregations of rules generate outcomes.

3. Outcomes and classes of outcomes can be path dependent or dependent on initial conditions.

4. The extent of diversity, rate of adaptation, connectedness and interdependence determines the complexity. Systems with moderate amounts of these attributes produce complexity.

5. Complex systems can vary in their robustness, their ability to maintaining functionality due to internal dynamics, entry and exit, and environmental changes.

In sum, we have highlighted five insights that have implications for the study of comparative politics. They may also lead to productive dialog between the two camps. The first, second, and fourth insights challenge the neoclassical approach to institutional analysis which does not address how equilibria are attained (Diermeier and Krehbiel 2003). Writing down the game, solving for the equilibrium, calculating comparative statics and testing for significants ignore the possibility that the system might instead head off on some complex path. Recall from Fig. 3 how the same model in different environments might produce either an equilibrium or complexity. We need to know how fast actors adapt, the diversity of types, and the interdependence (the fourth insight) of their behaviors before leaping to the assumption of an equilibrium. 
The third insight, the potential for complex systems to produce path dependent outcomes, implies that the sequencing of institutions can matter. This is not a new idea (Pierson 2004). What complexity theory adds are formal tools to classify and measure path dependence (Page 2006), to differentiate between path-dependent events and path-dependent distributions, and to differentiate path dependence from sensitivity to initial conditions (Lamberson and Page 2012).

The fifth insight, the distinction between robust and stable, opens up new questions. How does the creation of a new institution depend on or affect behavior in existing institutions? If new institutions can alter how people play in existing settings then path dependence may be mitigated (Bednar et al. 2012).

\section{Modeling the Interaction Between Institutions and Culture}

In Sect. 2, we described a persistent challenge in comparative politics research: how to account for differences in institutional performance when the institutions are embedded in different cultural contexts. Whether the institution is as focused as a set of rules to manage a common resource or as broad as a new constitution, identical institutions may not shape behavior identically. Our field has ample evidence that culture affects institutional performance, but we do not have a theory-one that is transportable across contexts - for the mechanism by which culture alters the way that agents respond to the information and incentives that institutions contain.

Our aim in constructing a model to capture the effects of culture was to include many of the hallmarks of cultural behavior. Culture describes common behavioral tendencies within a community, and distinctions between them, such as different ways of greeting friends in the U.S. and Britain — with a handshake-as opposed to the French, who tend to greet one another with light kisses on the cheek. Culture is often also invoked to explain deviations from predicted behavior, such as settling on suboptimal behavior or sticking with inefficient practices despite being exposed to more efficient methods.

To build a model capable of capturing these manifestations of culture, in a series of papers, and working with numerous collaborators, we have constructed a framework for modeling institutions in context. Our framework combines features of rational choice models and complex system models. From rational choice, we model institutions as game forms. We assume a set of actors, a set of possible actions, and payoffs from the actions. However, unlike many rational choice models, we do not assume that the actors take optimal actions. Instead, we endow agents with a behavioral repertoire that includes the capacity to take optimal actions along with a learning rule that they use to choose actions. We then set the model in motion, "growing" behavioral outcomes, in the language of the generative social science introduced by Epstein and Axtell (1996). ${ }^{12}$ Agents might find the

\footnotetext{
12 Epstein and Axtell open their book with this remark: "Perhaps on day people will interpret the question, 'Can you explain it?' as asking 'Can you grow it?"' Their book was a call to arms. If agents do not ever behave the way that social science models predicted, in what sense are these models explanatory?
} 
equilibrium assumed by game theory, but they may not. When outcomes vary from game-theoretic predictions, the behaviors depend on the path or they could depend on context. We consider both.

\subsection{Culture as Behavioral Spillovers}

In our framework, individuals participate in multiple institutions simultaneously (Bednar and Page 2007). As a benchmark, we first constructed several games that included the Prisoner's Dilemma and variations on it. For example, one variation is Alternation, where the payoff-maximizing behavior requires players to alternate their actions so that outcomes flip between diagonal cells. When players do this, they in effect take turns "winning", getting a low payoff and then a high payoff in alternating periods. In another game, players have a dominant strategy; while their payoffs are interdependent, their choice of action is not.

When our agents played any single game they nearly always learned the efficient equilibrium, in agreement with the rational choice models. However, when we introduced multiple games simultaneously, behaviors changed significantly. In the multiple game environment, agents learned actions for both games. The agents had the capacity to evolve different strategies for each game or to apply strategies learned in one game to the other. Contrary to the assumptions of rational choice models, often the agents no longer found the efficient equilibria. Instead, they produced outcomes that exhibited contextual sensitivity. The agents displayed patterns of behavior that we refer to as culture.

In the study by Bednar and Page (2007), we used both mathematical models and simulated interactions between computational agents. The simulation results generated culturally influenced behavioral tendencies in line with the conventional intuitions of culture's effect on political and economic interactions (see Box below). As would be expected in a complex system, we found that any two runs of the model might produce different outcomes. This resulted from a combination of sensitivity to initial conditions and path dependence. The implication for comparative politics should be clear: two populations who interact in identical institutions may learn different behaviors. We also found similar behavior across distinct games. If agents cooperated in one game, they were more likely to cooperate in another. Thus, the model produced cooperative cultures and selfish cultures, exhibiting inter-agent consistency across games, inter-agent coordination, and population differences between otherwise identical runs. 


\section{Features of Culturally-Affected Behavior}

(Bednar and Page 2007)

Intra-Agent Consistency: An individual's behavior in one setting correlates with, predicts, or shares features with her behavior in other settings.

Inter-Agent Coordination: Within a population, individuals coordinate on a common or a set of common actions.

Population Differences: Populations may coordinate on different common actions owing to path dependence.

Suboptimal Behavior: Populations may learn and coordinate on a suboptimal behavior.

Behavioral Stickiness: Individuals and populations may not respond to new incentives. Old, inefficient behaviors may be self-reinforcing.

Furthermore, we found that agents could locate equilibria-they settled into regularized behavior-if we lowered the rate of experimentation to almost zero. Small amount of experimentation produced substantial heterogeneity, an observation we return to later. The model, to an extent, supports the equilibrium behavior. A more accurate assessment would be that behavior resembled a cloud centered on an equilibrium, and that the equilibrium selected was often suboptimal because of the emergent consistency. Finally, we found the systems exhibited behavioral stickiness. Increasing incentives to produce efficient behaviors had limited effect: cultural behavior-dominated incentives.

The key insight from that first paper is that behavioral spillovers could be a mechanism by which culture influences institutional performance. If behavior in one game influenceS behavior in another, the result will be ensemble effects on institutional performance. Considering each institution in isolation loses predictive power.

Culture, defined as patterns of behavior, is a product of intra-agent consistency across games-agents carrying behaviors over from one game to the next. The diffusion of that carried-over behavior through the population results from interagent coordination. If an analyst were to look only at the institutions-even at multiple institutions-but ignore the coherence in behaviors generated by the spillovers, then an analyst would miss a key determinant of institutional performance. This first model shows that a complex systems approach could include a cultural component. 


\subsection{Behavioral Spillovers: The Direction of Influence}

The broader framework - the idea of repertoires applied to ensembles of institutions-proposes a new way to think about the relationship between institutions and understanding culture as patterns of behavior. Our initial analysis uses mathematical modeling to describe the effects of cognitive limitations, and computer simulations of purposive agents to generate behavior. Our response and that of those who engage our ideas as that a logical next step was to move beyond theory: to test our framework with human subjects. We chose an experimental approach so that we could control the context.

With collaborators Yan Chen and Tracy Liu we ran experiments to see how human subjects would respond in situations where they played multiple strategic games simultaneously (Bednar et al. 2012). Like the agents in our computational simulations, our human subjects repeatedly played games related to the Prisoner's Dilemma, including the dominant strategy game and two versions of an alternation game, in which the optimal equal payoff strategy involves each player allowing the other to win half of the time.

We found strong evidence of behavioral spillovers that aligned with the computational results. Subjects' responses to incentives depended on the other games they faced at the same time. Players developed consistent patterns of behavior. To distinguish between behavioral spillovers-where one game causally affects response to another-from mere cognitive overload, we made a prediction about the flow of the spillover. We hypothesized that subjects would settle on behavior for games that are easier to solve first and then apply that behavior to the games that are more difficult to solve.

Our hypothesis about the direction that behavioral spillovers would flow, from easy to difficult games, required us to develop a measure of game difficulty. We used the subjects' behavior in single-game studies to measure the entropy of outcomes. Entropy captures the uncertainty of play. Minimal entropy corresponds to everyone choosing the same action. Maximal entropy corresponds to players choosing actions randomly with equal likelihood.

In our lowest entropy game, nearly all subjects played the game in the same way. This game had a dominant strategy unique equilibrium. It was easy to solve. In highentropy games, players responded differently and they continued to learn. This was evidenced by analyses of individual players who changed his or her response over successive periods. We captured this statistically by linking patterns of play to specific strategies and showing that the maximum likelihood strategy changed over time.

Entropy, a statistical measure of surprise, proved to be a useful measure of predicting behavioral spillovers. If subjects were asked to play two games, one of high entropy and one of low entropy, they tended to play the low-entropy optimal response in the high-entropy game as well. For example, subjects playing the Prisoner's Dilemma, a high-entropy game, defected much more frequently when they also played a game that rewarded defection. When playing the Prisoners's Dilemma with a game that rewarded alternation, they alternated more often in the Prisoners' Dilemma game. 
This last behavior merits emphasis. When playing the Prisoners' Dilemma (PD) with an alternation game, subjects played a much more complicated strategy in the PD: they took turns between getting the "temptation" and the "sucker's payoff", a behavior never seen when they played the PD alone. The alternating strategy also produced a lower payoff. Behavioral spillovers lead to suboptimal performance, and in a direction that is predictable and resembles a cultural influence.

In our analysis, we interpreted game entropy to be a measure of game difficulty, but other interpretations of entropy connect to important questions in comparative politics. Entropy, again, is a measure of the unpredictability of responses that subjects have to identical information and incentives. Higher entropy means that people respond differently to the same influences. Low-entropy means that they tend to respond identically. While one might think that the most interesting institutions are those that produce high entropy (and we do make an argument for their usefulness below), institutions with low entropy demand inspection: why does everyone conform to the same behavior? The influence of role norms, a religion with strict rules of conduct, or overwhelming incentives, whether pleasurable or painful, drive behavior toward uniformity. Often, even identifying the conditions that create low entropy requires specific localized knowledge. And once identified, those forces may affect behavior outside of their immediate context.

Our framework implies that behavioral constraints imposed by social roles, religion, or a police state might extend beyond the immediate realm of that force's influence. Religious faith may shape political expression; female subservience within the family may shape political expression, economic activities, and even classroom dynamics. Though hesitant to push too far, we believe that a portion of the within-country coherence evident in the World Values Surveys and well understood by area studies specialists may be explained through spillovers. We see that as an empirical question worth exploring.

\subsection{Institutional Path Dependence}

One of the most important roles of comparative political science is to help design and advise developmental projects. These projects aim to create more equitable societies, thriving economies, and more environmentally sustainable practices. Not only are comparative political scientists called upon to make recommendations about ideal political or economic institutions, but to say how one might effectively transition from the status quo to the idealized state. While our field has generated predictions, based upon empirical regularities, of what conditions lead to democratization or autocratization, ${ }^{13}$ we are less confident in recommending pathways toward a goal. For example, following the collapse of the Soviet Union, the newly independent political economies of eastern Europe sought advice for how to transition to democracies and open market economies. Equally compelling arguments recommended paths that clashed head on: big bang vs gradualism (eg. Dewatripont and Roland 1992; Lipton and Sachs 1990). Without a theory of the

\footnotetext{
13 See, for example, O’Donnell and Schmitter (1986), Acemoglu and Robinson (2001), Boix (2003).
} 
mechanism for how the cultural context would affect people's response to institutions, our field was left to argue with itself.

While the science remains preliminary, with an adaptive model of the interaction between institutions and culture in hand one might begin to make headway on this key problem. The methodology that we propose of behavioral spillovers my help to chart optimal paths, recommending intermediate steps that should be taken to reach social goals. To explore whether that might be possible in Bednar and Page (2015) we adapted our model of multi-institutional play to characterize the optimal sequencing of institutions. We designed a set of games that vary parametrically, creating a space of relatedness. In the most intuitive form, imagine a set of $2 \times 2$ games where the payoffs include one parameter. As that parameter is increased incrementally, the payoffs in the cells change, and therefore, is a different institution. The institutions are related spatially by the parameter; those with more similar values of the parameter are more alike. The games (or institutions) can then be arrayed along a single dimension capturing their similarity to one another.

Using this measure of relatedness, we identify institutions that are immune from behavioral spillovers - the influence of other games present in society-and those that are vulnerable or susceptible to influence. In a unidimensional space, the susceptible games are those in the middling range, and games with parameter values toward the limits of the range are immune-meaning that all agents will play the game in the same way, and optimally. Unlike the analysis in Bednar and Page (2007) where actors played games simultaneously, in this analysis we introduce the institutions sequentially. We also posit two types of agents who differ in the way of their initial response to a new institution. Some will behave like good game theorists; they consider only the institution at hand, and identify and play the payoffmaximizing strategy, at least initially. A second type relies on its repertoire. It makes an educated guess, applying the behavior that it used in the game that it has played that is most similar to the new problem at hand. (We assume that for the very first round of games, everyone behaves like a game theorist.)

Both types of agents then respond to their environment. Outcomes emerge from the interactions of the agents. We find both set dependence (the set of institutions matter) and path dependence. We even find that the introduction of some games biases outcomes in other games toward better equilibria. For example, learning how to coordinate in alternation games can be facilitated by having agents first play the Stag Hunt game.

Clark (1997) reminds us, "nature is heavily bound by achieved solutions to previously encountered problems" (p. 81). Thus, when choosing or designing an institution or an institution, we should not limit attention to the equilibria it implements. We ought also consider existing individual and collective behavioral repertoires, and take into account their effects on institutional performance, choice, and even design. Our next result connects institutional path dependence to social potential. 

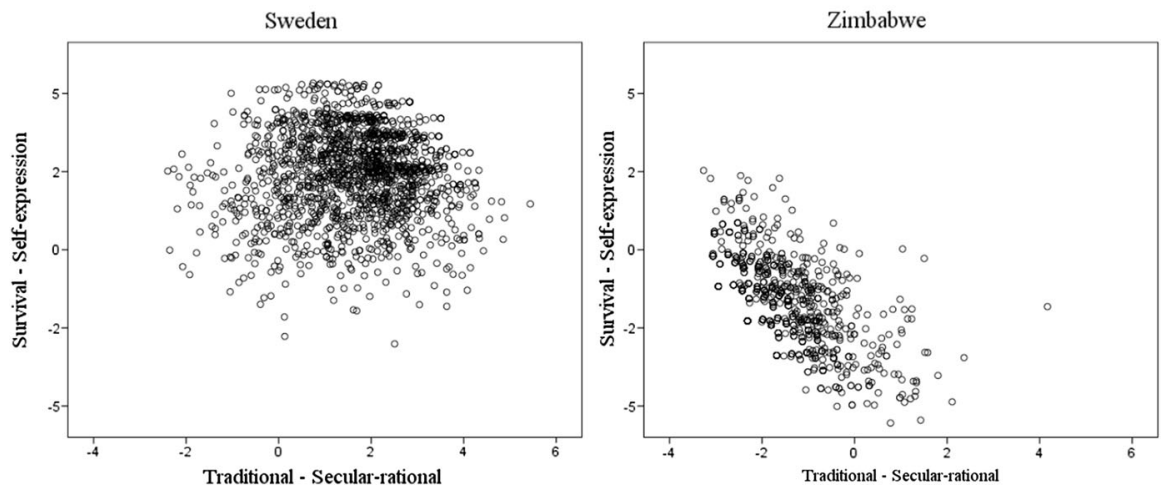

Fig. 4 Between- and within-country heterogeneity in world values survey

\subsection{Limiting Paths and Behavioral Diversity}

A sequential analysis demonstrates how existing behaviors-cultures-limit the capacity of a population to adapt to new circumstances. In Bednar, Jones-Rooy, and Page (2015), we found that games differed in the extent to which they led to inefficient outcomes in subsequent games. Some games, most notably games in which one type of player learned to defer to another type, induced behaviors that became locked-in for future games. For example, under some conditions, hierarchical populations will continue with hierarchical societies, rather than choose an institution that distributes payoffs more equitably. We refer to these behaviors as path limiting.

In the paper, we compare games by the extent to which they limited future paths. Our key finding is that populations that maintain behavioral diversity are better able to adapt than those that do not. Populations with more diversity behaviors possess larger adaptive capacity. They are able to learn more behaviors. A population that supports cooperation in the Prisoners' Dilemma game throughout both Grim Trigger and Tit for Tat can quickly learn both selfish behavior (by moving to the punishment state of Grim Trigger) and alternation (by playing Tit for Tat but starting in different states). In contrast, a population that only knows how to play selfishly may have difficulty learning how to alternate or cooperate. Behavioral diversity, therefore, contributes to the robustness of the system.

To apply our theories to real-world cases, one must be able to identify relationships between games: relative difficulty or ease, or strategic familiarity. Application of the theory requires the expertise of someone familiar with cultural communities who can make these connections. This enterprise is quite feasible, and demonstrated in the aforementioned work of Henrich and his many collaborators.

\subsection{Modeling Diversity within Cultures}

As we continued to think about how to capture the interaction between institutions and culture, we were drawn to a paradox of culture: it is coherence and internal 
diversity. In each of the simple games we studied, actors benefitted from coordinating their behaviors. Analytically, one might expect that given enough time to interact, all actors would converge on a single set of behavioral rules.

In our computational models, the agents did not converge. That was fortunate for us. Complete convergence is at odds with reality. While cultures are coherent enough to be identifiable from one another, no individual has a culture, and no two individuals within a cultural community always behave identically, regardless of how cohesive the culture is. Culture is an emergent phenomenon, a product of the social interaction between individuals who are different from one another when viewed at close range, but appear similar when viewed from a coarser lens. Our colleague Ron Inglehart provided us with data from the World Values Survey to illustrate the internal diversity within distinct cultural communities (Fig. 4).

Our model produces similarly cloudy pictures. Given that we assumed small rates of experimentation, we expected some variation. Yet, the radii of our clouds were orders of magnitude larger than we expected. To understand within-population heterogeneity, we opted to write a minimalist model. We focus on two causal forces: a desire to coordinate actions with others and a desire to be internally consistent across games (Bednar et al. 2010). These two incentives could align: an individual who cooperated in most settings could be embedded in a cooperative population. More cooperative actions would then increase both consistency and cooperation.

In the model, we first we analyzed the influences of consistency and coordination alone. We found no amplification: the level of heterogeneity scales with the level of experimentation. In the full coordination-consistency model, we found that even small rates of experimentation amplified to produce persistent and substantive heterogeneity. Using a Markov model, we were able to show how the two forces together create a complex dynamic process that maintains diversity.

This result, like the earlier finding that considering multiple games influences outcomes, provides formal justification for comparativists' claims that stark gametheoretic models can miss the mark. When we consider multiple games or multiple incentives, we get different results. When actors play multiple games, they may no longer learn efficient equilibria. When they make small errors in contexts with multiple forces, those errors may magnify to produce sustained heterogeneity.

Our complex systems approach takes two steps that bridge rational choice and thick description. We include multiple institutions and we assume purposive behavior. These two substantial steps toward greater realism do not force us to abandon the power of formal analysis. Taken together they allow us to generate consistent yet heterogeneous behavior within cultures, variation across cultures, coordination on inefficient equilibria, and behavioral stickiness. They also relate the proper sequencing of institutions to the development of behavioral repertoires. Whether these qualitative properties can be empirically linked to actual differences seems to us at least worthy of enquiry. 


\section{Discussion}

Our multi-institutional framework uses assumptions, ideas, and tools from complex systems to address a key question in comparative politics: how institutions and culture affect one another. Complex systems methods do not substitute for game theory or thicker descriptive accounts, but instead are complementary analytical tools that can build bridges between the existing silos and challenge the core beliefs of their inhabitants For those reasons, we believe a complexity approach can improve the discipline by providing lattices upon which scholars can array descriptive accounts yet maintain a commitment to logical causality. If so, the inclusion of complex systems approaches into the study of comparative politics may move us closer to an understanding of the potential for one-size-fits-all models as well as the extent of exceptionalism.

Our research focuses on cognitive repertoires: how they emerge, their properties, how they influence the performance of institutions, and, by extension, how they can advantage some institutional designs and choices over others. We view our results as evidence that a complex systems approach may help explain variation in institutional performance. We expect that future complex system approaches will include network structures, identity groups, and other relevant features of social systems. One extension we plan to pursue in the near term allows for social enforcement. $^{14}$

Here, we sketch how some of these other features might be included individually and jointly and their potential importance. First, network structures emerge. Constitutions may define roles and rules, but who interacts with whom will be the result of some adaptive process. The resultant network structures can influence how and whether information aggregates (Jackson 2008). They also limit and create power. A person's position in a network contributes to her ability to achieve desired political outcomes (Burt 1995).

Notice the parallels between networks and behavioral ensembles. Both emerge as actors learn and adapt within political institutions. Both shape and influence the outcomes that can be achieved. Both could produce institutional path dependence. We described how institutions could create behaviors that advantage some institutions over others.

A similar phenomenon can occur for networks. An institution could cause a network to emerge that makes some future institutions more efficient than others. Relatedly, the network may function poorly or efficiently at some other tasks. Ben and Jackson (2010) derive conditions for networks to converge on a collective prediction. The collective intelligence of a community (Elster and Landemore 2014), therefore, depends on the network structure. That structure may depend on institutional ensembles. Finally, the diversity maintained within a society can also depend on the network structure (Economo et al. 2016). Diversity, as well, contributes to collective intelligence (Page 2008).

\footnotetext{
14 Thank you Rob Boyd for suggesting that we make this modeling extension a priority. In the meanwhile, we refer readers to Axelrod (1986).
} 
The effects of including identity take a different form. Some identity classifications-nationalities, religions, and gender-will be exogenous. Others can emerge. These identity effects can interact with behavioral ensembles. Individuals may choose behaviors that align with their identities. Or they may learn to act differently within and between identity groups (Riolo et al. 2001). Identities can also interact with network structures. Individuals may be more likely to make connections with others who belong to the same identity groups. The resulting homophily may produce behaviors and information that vary by identity group.

If we put everything together: behavior, networks, and identities, we move closer to a realistic model. We advise not jumping immediately to realistic models as good model building often appends features models with known implications (Miller and Page 2007). Thus, the appropriate path may be to add networks and identities singly to the behavioral repertoire models before combining all three.

Ruth Lane wrote in her excellent pitch for the capacity of agent-based modeling - a method within complexity science-to enhance comparative politics research: "Axelrod (2006) has argued that bottom-up models can unify different scientific disciplines; it may be that such models can also assist in building bridges between parts of a single field that have been too long in opposition. A collaboration between those, in political science, who rejoice in the plenitude of facts, and those who lust after an appropriate degree of scientific rigor is one of the interesting possibilities inherent in bottom-up analysis" (Lane 2016:1146). We could not say it better.

\section{References}

Acemoglu, Daron, and James A. Robinson. 2001. A theory of political transitions. American Economic Review 91(4): 938-963.

Axelrod, Robert. 1986. An evolutionary approach to norms. American Political Science Review 80(4): 1095-1111.

Axelrod, Robert. 2006. Agent-based modeling as a bridge between disciplines. In Handbook of computational economics, vol. 2, ed. L. Tesfatsion, and K. Judd. Amsterdam: Elsevier.

Bates, Robert H., Avner Greif, Margaret Levi, Jean-Laurent Rosenthal, and Barry R. Weingast. 1998. Analytic narratives. Princeton: Princeton University Press.

Bednar, Jenna. 2009. The robust federation: principles of design. New York: Cambridge University Press.

Bednar, Jenna, Scott E. Page, and Jameson Toole. 2012. Revised-path dependence. Political analysis 20(2): 146-156.

Bednar, Jenna, Aaron Bramson, Andrea Jones-Rooy, and Scott E. Page. 2010. Emergent cultural signatures and persistent diversity: a model of conformity and consistency. Rationality and Society 22(4): 407-444.

Bednar, Jenna, Yan Chen, Tracy Xiao Liu, and Scott E. Page. 2012. Behavioral spillovers and cognitive load in multiple games: an experimental study. Games and Economic Behavior 74(1): 12-31.

Bednar, Jenna, Andrea Jones-Rooy, and Scott E. Page. 2015. Choosing a future based on the past: institutions, behavior, and path dependence. European Journal of Political Economy 40(B): 312-322.

Bednar, Jenna, and Scott E. Page. 2007. Can Game(s) theory explain culture? The emergence of cultural behavior within multiple games. Rationality and Society 19(1): 65-97. 
Bednar, Jenna, and Scott E. Page. 2015. When order affects performance: institutional sequencing, cultural sway, and behavioral path dependence. Michigan: University of Michigan manuscript.

Ben, Golub, and Matthew O. Jackson. 2010. Naive learning in social networks: convergence, influence and the wisdom of crowds. American Economic Journal: Microeconomics 2(1): 112-149.

Boix, Carles. 2003. Democracy and redistribution. New York: Cambridge University Press.

Boyd, Robert and Peter J. Richerson. 1988. Culture and the evolutionary process. Chicago: University of Chicago Press.

Boyd, Robert and Peter J. Richerson. 2005. The origin and evolution of cultures. Oxford: Oxford University Press.

Burt, Ronald. 1995. Structural holes: the social structure of competition. Cambridge: Harvard University Press.

Clark, Andrew. 1997. Being there: putting brain, body, and world together again. Cambridge: MIT Press.

Clark, William R., Matt Golder, and Sona Golder. 2012. Principles of comparative politics. Washington, DC: Sage: CG Press.

Dewatripont, Mathias, and Gérard Roland. 1992. The virtues of gradualism and legitimacy in the transition to a market economy. The Economic Journal 102(411): 291-300.

Diermeier, Daniel, and Keith Krehbiel. 2003. Institutionalism as methodology. Journal of Theoretical Politics 15(2): 123-144.

Economo, Evan, Lu Hong, and Scott E. Page. 2016. Social structure, endogenous diversity, and collective accuracy. Journal of Economic Behavior 125: 212-231.

Elster, Jon, and Hélène Landemore. 2014. Collective wisdom: principles and mechanisms. Cambridge: Cambridge University Press.

Epstein, Joshua M., and Robert L. Axtell. 1996. Growing artificial societies: social science from the bottom up. Washington, DC: Brookings Institution Press.

Falleti, Tulia G., and Julia F. Lynch. 2009. Context and causal mechanisms in political analysis. Comparative Political Studies 42(9): 1143-1166.

Ferejohn, John. 1991. Rationality and interpretation: parliamentary elections in early Stuart England. In The economic approach to politics: a critical rassessment of the theory of rational action, ed. Kristen R. Monroe, and Anthony Downs. New York: Addison-Wesley.

Greif, Avner. 2006. Institutions and the path to the modern economy. Cambridge: Cambridge University Press.

Greif, Avner, and David Laitin. 2004. A theory of endogenous institutional change. American Political Science Review 98: 633-652.

Henrich, Joseph, Robert Boyd, Samuel Bowles, Colin Camerer, Ernst Fehr, Herbert Gintis, and Richard McElreath. 2001. In search of homo economicus: behavioral experiments in 15 small-scale societies. The American Economic Review 91(2): 73-78.

Jackson, Matthew O. 2008. Social and economic networks. Princeton: Princeton University Press.

Jervis, Robert. 1998. System effects: complexity in political and social life. Princeton: Princeton University Press.

Johnson, Neil. 2009. Simply complexity: a clear guide to complexity theory. London: Oneworld Publishing.

Lamberson, P.J., and Scott E. Page. 2012. Optimal forecasting groups. Management Science 58(4): 805-810.

Lipton, David, and Jeffrey Sachs. 1990. Creating a market economy in Eastern Europe: the case of Poland. Brookings papers on economic activity 1990(1): 75-133.

Lane, Ruth. 2016. Sartori's challenge: political models from the bottom up. Comparative Political Studies 49(8): 1128-1160.

Lijphart, Arend. 1984. Democracies: patterns of majoritarian \& consensus government in twenty-one countries. New Haven: Yale University Press.

Lijphart, Arend. 1999. Patterns of Democracy: government forms \& performance in thirty-six democracies. New Haven: Yale University Press.

Lizardo, Omar, and Michael, Strand. 2010. Skills, toolkits, contexts, and institutions: Clarifying the relationship between different approaches to cognition in cultural sociology. Poetics 38: 204-227.

Mahoney, James, and Kathleen Thelen. 2009. Explaining institutional change: ambiguity, agency, and power. New York: Cambridge University Press.

Medin, Douglas, Will Bennis, and Michael Chandler. 2010. Culture and the home-field disadvantage. Perspectives on Psychological Science 5(6): 708-713.

Miller, John H. 2016. A crude look at the whole. New York: Basic Books. 
Miller, John H., and Scott E. Page. 2007. Complex adaptive systems: an introduction to computational models of social life. Princeton: Princeton University Press.

Mitchell, Melanie. 2009. Complexity: a guided tour. Oxford: Oxford University Press.

Newman, Mark E.J. 2005. Power laws, Pareto distributions and Zipf's Law. Contemporary Physics 46(5): 323-351.

Newman, Mark E.J. 2010. Networks: an introduction. Oxford: Oxford University Press.

Nisbett, R.E., K. Peng, I. Choi, and A. Norenzayan. 2001. Culture and systems of thought: holistic versus analytic cognition? Psychological Review 108: 291-310.

O’Donnell, Guillermo, and Philippe C. Schmitter. 1986. Transitions from authoritarian rule: tentative conclusions about uncertain democracies. Baltimore: Johns Hopkins Press.

Oosterbeek, Hessel, Randolph Sloof, and Gijs Van De Kuilen. 2004. Cultural differences in ultimatum game experiments: evidence from a meta-analysis. Experimental Economics 7: 171-188.

Page, Scott E. 2006. Essay: path dependence. Quarterly Journal of Political Science 1: 87-115.

Page, Scott E. 2008. The difference: how the power of diversity creates better groups, firms, schools, and societies. Princeton: Princeton University Press.

Page, Scott E. 2010. Diversity and complexity. Princeton: Princeton University Press.

Page, Scott E. 2015. What sociologists should know about complexity. Annual Review of Sociology 41: $21-41$

Pierson, Paul. 2004. Politics in time: history, institutions, and social analysis. Princeton: Princeton University Press.

Putnam, Robert D. 1988. Diplomacy and domestic politics: the logic of two-level games. International Organization 42(3): 427-460.

Riolo, Rick, Michael D. Cohen, and Robert Axelrod. 2001. Evolution of cooperation without reciprocity. Nature 414(22): 441-443.

Schmitter, Philippe C. 2009. The nature and future of comparative politics. European Political Science Review 1(1): 33-61.

Swidler, Anne. 1986. Culture in action: Symbols and strategies. American Sociological Review 51(2): 273-286.

Thelen, Kathleen. 1999. Historical Institutionalism in comparative politics. Annual Review of Political Science 2(1): 369-404.

Tsebelis, George. 1990. Nested games: rational choice in comparative politics. Berkeley: University of California Press.

Wolfram, Stephen. 2002. A new kind of science. Champaign: Wolfram Media Inc. 